\title{
The Fuzzy and Dynamic Nature of Trust
}

\author{
Chang E, Thomson P, Dillon*, TS, Hussain FK \\ Centre for Extended Enterprise and Business Intelligence \\ Curtin Business School, Curtin University of Technology, Perth, Western Australia \\ Elizabeth.Chang, Patricia.Thomson, Farookh.Hussain@cbs.curtin.edu.au \\ * Faculty of Information Technology \\ University Technology Sydney, Sydney, Australia \\ Tharam@it.uts.edu.au
}

\begin{abstract}
Trust is one of the most fuzzy, dynamic and complex concepts in both social and business relationships. The difficulty in measuring Trust and predicting Trustworthiness in service oriented network environments leads to many questions. These include issues such as how to measure the willingness and capability of individuals in the Trust dynamic and how to assign a concrete level of Trust to an individual or an Agent in a service oriented environment. It is also necessary to understand why Trust is fuzzy and changing with time and why we need to make sure that the context is clear when we refer to a Trust Relationship between two Agents.

The dynamic nature of Trust creates the biggest challenge in measuring Trust and predicting Trustworthiness. In order to develop a Trustworthiness Measure and Prediction Method, we first need to understand what we can actually measure in a Trust Relationship. In this paper, we analyze the fuzzy, dynamic and complex nature of Trust. Note that we use the term fuzzy in this paper not in the sense of the precise definitions given in the Fuzzy Systems literature, but to indicate a certain vagueness, complexity or inappropriate definition and qualitative characterization, rather than a quantitative representation.
\end{abstract}

Keywords: Fuzzy, Dynamic and Complex nature, Implicitness, Asymmetry, Transitivity, Antonym, Asynchrony, Gravity, Endogenous impact, Exogenous impact, Psychological factors, Time spot, Timeslot, Time space, Managing the fuzzy, dynamic and complex nature of trust

\section{Introduction}

Trustworthiness Measurement and prediction are complex and limited by the fuzzy, dynamic and complex nature of Trust. In this context, we need to consider the social aspects of fuzziness, dynamism and the complexity of Trust. Some explicit considerations are relevant to this:

- The term fuzzy refers to the indefinite or imprecise and sometimes unclear nature of Trust.

- The term dynamic refers to Trust not being stable or always changing as time passes.

- The term complex refers to the multiple ways of measuring and the variety in views on Trust.

We note that when something cannot be explicitly defined, and is not stable and associated with a variety of views and opinions, it always difficult to manage and predict.

\section{$2 \quad$ Existing Literature}

Upon reviewing the existing literature on Trust, it is evident that there have not been many studies into the fuzziness, dynamism and complexity of Trust of the impact on Trust, Trust Measurement and Trustworthiness prediction, especially in the world of e-business and in service oriented network environments.

Some studies by Egger (including those of Shelat and Egger, 2002; Egger and Groot, 2000; Egger, 2000 and 2003) consider how the usability of Websites (a Website may represent a service provider), the way content is organized and how security and privacy issues are addressed, communicate Trust to their human users. Factors considered by Egger are applicable for B2C (Business to Customer) e-commerce, 
where the customer (usually the client) interacts with the service providers through websites. Kim and Moon (1997) investigated how graphic design elements in a website can communicate Trust to human users. However, the studies do not investigate how the usability of a Website can assist in communicating, establishing Trust between providers and customers and Trustworthiness Measurement and prediction. Other work only provides reference to a single Trust Value and a single context for Trust Management. They do not consider other factors such as context dependence, time slots for frames, or internal factors of interacting parties or Agents, nor have they examined all the possible fuzzy, dynamic and complex characteristics of Trust.

Psychological nature of the Trusting Agent has impact on the trust decisions to another Agent. In psychological terms, according to Myers (2003) and Mallach (2000), it is reasonable to assume that: People with a 'sensing preference' will not trust any person with whom they did not have any previous interaction. Both Myers and Mallach indicate that people with a 'Sensing' preference have a tendency to rely on facts and experience (Myers, 2003; Mallach, 2000). People with an 'intuition preference' may trust a person with whom they have not had any previous interactions. The preference of the Trusting Agent will influence its decision to trust a given Trusted Agent, with or without detailed information on the trustworthiness of the Trusted Agent. Myers and Mallach contend that persons with an 'Intuition' preference have a tendency to rely more on possibilities and taking risks (Myers, 2003; Mallach, 2000). People with a 'thinking preference' have a tendency to analyse things in an objective and logical fashion with little or no regard for personal values before they reach or take a decision (Myers, 2003; Mallach, 2000). We could also believe that if the Trusting Agent has a thinking preference, he/she will pay little or no attention to the personal values of the Trusted Agent, or to personal feelings about the Trusted Agent and make an objective and logical decision regarding whether to trust the Trusted Agent or not. People with a 'feeling preference' place primary importance on personal values, before reaching a decision (Myers, 2003; Mallach, 2000). We could also believe that the Trusting Agents who give preference to feeling will place greater importance on his/her personal feelings about the Trusted Agent and values of the Trusted Agent while they decide whether or not to trust the Trusted Agent [2,7].

\section{$3 \quad$ Fuzzy and Dynamic Characteristics of Trust}

The six important fuzzy and dynamic characteristics of Trust are the Implicitness in Trust, Asymmetry in Trust, Transitivity in Trust, Antonymy in Context, Asynchrony in Time Space and Gravity in Relationships. These factors create big challenges in Trustworthiness measurement and prediction. They are important to the understanding of the complexity of Trust and its measurement and prediction.

\subsection{Implicitness}

Trust is implicit. This means that a Trusting Agent may not be able to explicitly specify their belief, the willingness and capability of the Trusted Agent, and the context and the time dependency of Trust. These can only be estimated. A Trust Relationship can involve one individual only (i.e. "I Trust myself"), or involve another party or Agent (i.e. I Trust my boss), or a group or an organisation (i.e. I Trust the Bank). We can most often define the context and time frame relating to a Trust Relationship, but we cannot explicitly state the willingness and capability of individuals or others involved in the Trust Relationship; nor the understanding that the context may change and as time passes, beliefs change. Trust is therefore implicit (i.e. understood by parties). Trust is fuzzy because it is not obviously stated. Trust is also dynamic as individuals may able to define the 'context' and 'time slot' relating to Trust but they cannot give explicit definitions of 'willingness' and 'capability' with regard to an individual or others about their Trust. This reinforces the view that Trust is implicit. The only thing we can do is to give an estimate of 'willingness' and 'capability', through behaviour monitoring, evaluation and a correlation with an individual's behaviour. The challenge in Trustworthiness Measurement or Trustworthiness Prediction is the degree of the implicitness of Trust ; that is the explicit measure of 'belief', "willingness' and 'capability' in the Trust Dynamic. We can provide an estimate of this measure through a well known scientific method; namely the correlation or regression of behaviour or a correlation between what people say and what people do. In business we can correlate committed services with an actual delivered service to validate the Trust level. 


\subsection{Asymmetry}

Trust is asymmetric. This means that a Trusting Agent has a certain belief in the Trusted Agent in a particular context. It does not imply that the Trusted Agent 'B' should have the same belief in the Trusting Agent ' $\mathrm{A}$ ' in the same context. Hence, due to the non-mutual reciprocal nature in the Trust Relationship, Trust is asymmetric. The characteristics of the Trust Relationship are also influenced by the Agents' internal factors (characteristics). There is no explicit understanding of the value of the Trust in the relationship between the two parties unless it is, in a human context, verbalised. In general terms, Agents do not explicitly verbalise a numeric Trust Value; they generally verbalise a level of Trust. Fuzziness, therefore, is evident. Trust can change from being symmetric to asymmetric. Let us assume that Agent A and Agent B Trust each other to exchange or deliver high quality music to each other in 2004. With the passage of time in 2005, Agent B's capability or willingness to deliver high quality music to Agent A decreases. As a result of this, the Trust that Agent A has in Agent B in the context of procuring or delivering high quality music decreases or becomes null. Hence we see that Trust, which was initially symmetric (equal) between two Agents, has become asymmetric due to the passing of time. This is also related to the dynamic nature of Trust with time. Trust is uni-directional (goes in one direction).

This means that if we assume the Trusting Agent A to be Alice and the Trusted Agent B to be Bob, the Trust Measure or estimation is only from Alice to Bob or Bob to Alice, but not both. However, Bob can also be a Trusting Agent and Alice is his Trusted Agent. We consider that this is a different Trust Relationship, because it has a different Trust Value. The Trust level and the Trust Value are assigned by the Trusting Agent to the Trusted Agent. It needs to be clearly understood that the Trust measure or prediction is asymmetric, regardless as to whether it is measured in the physical world or the virtual world. The challenge of a Trustworthiness Measure and prediction is conditioned on the asymmetric character of Trust. Therefore, one Trust Value does not represent both parties in a Trust Relationship. This is often implicitly assumed in a static social world which is conceptually negligent, as Trust in the social world can also imply a dynamic exchange between individuals that is sometimes multidirectional. A Trust Measure in a service oriented network environment must be uni-directional and only from a Trusting Agent to the Trusted Agent. It is only meaningful to the Trusting Agent and for use by the Trusting Agent. The Trust Value can move from symmetric to asymmetric or vice versa. Fuzziness and dynamism is therefore again apparent in the situation.

\subsection{Transitiveness}

Trust is transitive. It is illogical to assume that transitive Trust is an explicit phenomenon. The transitivity of Trust, also known as a derived Trust, means that Trust is derived from an existing Trust between Agents. Note that derived Trust and the Trust from which it is derived should be considered within the same context. It is important to understand that this derived Trust may be explicit, but generally, it is very hard to quantify accurately. We assume then, some level of implicitness (fuzziness). The level of Trust through a transitive introduction may be held at the same level between both parties and is dependent on the strength of the original Agent's Trust relationship. Transitive Trust is a very important concept in the service-oriented network environment where anonymous users or Agents often want to identify quality service through a transitive introduction, also known as "a recommendation" or "reputation". The recommendation or reputation is fuzzy in the sense that a transitive introduction is context and time dependent. These dependencies are not always explicit as there is an innate inability to hold the same view or understanding about the context and the exact time frame where the Trust Value or level was assigned.

Transitive Trust is also time dependent, which means that it depends on when a Trust Value was assigned and when this Trust Value was recommended. Trustworthiness prediction has to take aggregated time frames or slots in order to more accurately determine a Trust Value. Note that this value could change when time passes. This is the dynamic characteristic of Trust. Transitive Trust is affected by other opinions. The challenge of a Trustworthiness Measure and Prediction is the method of using a transitive Trust Value, also known as the recommendation value. Often we derive different Trust Values from 
different Agents about 'a particular Agent' or 'a service'. They relate to different time frames (time slots). Additionally, we have to consider first hand, second hand and third hand opinions.

\subsection{Antonymy}

The antonymous nature of Trust is related to "Context"; that is the Context may understood differently by two Agents, A and B, involved in a Trust relationship. Therefore, what may be clear to one Agent may not be clear to another. Fuzziness is evident in the antonymous dynamic of the Trust relationship. The context, as seen from the perspective of Agent A, may be the opposite or different from that seen from the perspective of Agent B. We note that the "context" may be understood in an opposite way; it is used in a different way and often implicitly recognized by either party or Agents. Each party perceives the context to be the opposite of that perceived by the other party. Agent A may see the context of the Trust relationship as one of "buy"; Agent B may see the context of the Trust relationship as one of "sell".

Fuzziness is evident because of the antonymous nature of the relationship between both Agents. The challenge of the Trust measure and the Trustworthiness prediction is to define the context clearly. This is difficult to do even in the real physical world.

\subsection{Asynchrony}

The asynchronous nature of Trust refers to asynchrony in a "Time Slot". That is the time slot of the Trustworthiness may be understood or defined differently between Trusting Agents and Trusted Agents. Fuzziness is inherent in any situation that becomes unclear to either party or Agents in the Trust relationship. Agents may understand the time slot differently for a given same context. The timeslots between the Agents may be the same, completely different, or partially overlapping. As a result of the asynchronous nature of timeslots, Trustworthiness prediction cannot be straight forward. The challenge of the Trust measure and Trustworthiness prediction is that we have to deal with different time slots in a time space. We need both to aggregate the time slot and also average the Trust Value over the aggregated time slots. This is important when recommendations or Trust reputation takes place.

\subsection{Gravity}

The Gravity of Trust refers to the gravity of the Trust relationship; the seriousness of the relationship to each Agent, or the influence on each party to the relationship. Each Agent has their own views on whether or not the relationship means much to them, and what influence it could have on their business or lives. As stated in all previous examples, fuzziness and dynamism is inherent in this characteristic. Regardless of who is the Trusting Agent or the Trusted Agent, from Agent A's point of view, the Agent A to Agent B relationship within a particular context could be unimportant to Agent A. However, from Agent B's point of view, the relationship may be very important.

\section{$4 \quad$ Reasoning the Fuzziness and Dynamism}

In the previous section, we illustrated the six characteristics of the fuzziness and dynamism of Trust. We also illustrated the endogenous and exogenous factors of the Agents. Now we would like to show how these are related to each other.

\subsection{Internal Factors of Trusted Agent}

In order to study why Trust is fuzzy and dynamic, we now look at the Agents who are involved in the Trust Relationship and their impact on the fuzziness and dynamism of Trust. In service oriented network environments, we have defined Trust as the belief that the Trusting Agent has in the Trusted Agent's willingness and capability to deliver a mutually agreed service in a given context and in a given time slot. The key challenge is how to measure willingness and capability, so that a Trust Value can closely represent the truth or quality of the Trusted Agents. 
Willingness symbolizes the Trusted Agent's will to act or be in readiness to act gladly, honestly, truthfully, reliably and sincerely in delivering on the mutually agreed behaviour. As this factor is internal to Agents, it is very hard to estimate even with scientific research methods. The willingness of a person or an Agent could change as time passes, as it may be dependent on the mood of a person. Therefore, it makes a Trust Model dynamic.

Capability is defined as "the talent, competence, aptitude, and ability of the Trusted Agent in delivering on the mutually agreed services”. Capability signifies the Agent's intelligence. It is internal to an Agent. A person or an Agent's intelligence changes with time due to internal or external influences. Examples of external influences could be further training or study. These changes could happen in any given time slot or over many time slots.

These two factors are internal factors of Agents. As they are internal, we therefore cannot have direct measures because we can not obtain it on hand to qualify it. Therefore, when we derive a Trust Value, it is only an estimate or an approximate value. Willingness symbolizes the Trusted Agent's will to act or be in readiness to act gladly, honestly, truthfully, reliably and sincerely in delivering on the mutually agreed behaviour. As this factor is internal to Agents, it is very hard to estimate even with scientific research methods. The willingness of a person or an Agent could be changed as time passes, as it could be dependent on the mood of a person. Therefore, it makes the Trust Model dynamic. We have defined Capability as the talent, competence, aptitude, and ability of the Trusted Agent in delivering on the mutually agreed service. Capability signifies an Agent's intelligence. It is internal to an Agent. A person or an Agent's intelligence changes with time due to internal or external influences. Examples of external influences could be further training or study. These changes could happen in any given time slot or over many time slots. These two factors are internal factors of Agents. As they are internal factors, we therefore cannot have direct measures because we can not obtain information to quantify the measure. Therefore, when we derive a Trust Value, it is only an estimate or an approximate value.

\subsection{Fuzzy and Dynamic Characteristics in Trust Model}

The six Fuzzy and Dynamic characteristics of Trust are as follows: Implicitness in Trust, Asymmetry in Trust, Transitivity in Trust, Antonymy in Context, Asynchrony in Time, and Gravity in Relationship. In view of the six fuzzy and dynamic characteristics of Trust, we could distinguish that characteristics 1 to 3 are related to Trust Value and characteristics 4 to 6 are related to Trust relationships.

- 'Implicitness of Trust ', 'Asymmetry in Trust' and 'Transitivity of Trust' are related to Trust Values because Agents make a decision on Trust Values, not anything else. Therefore 'implicitness', 'asymmetry', 'transitivity' are relevant only to Trust Values;

- 'Antonym in context', 'Asynchrony in Time Space' and 'Gravity of the Relationship' are related to the Trust Relationship, which is context and time dependent. Agents make the perception of the context, time or gravity of the relationship. This is an Agent's own view or opinion about what they see or believe in a Trust Relationship.

\subsection{Endogenous and Exogenous Characteristics}

In view of the Trust Model, we see the relationship involves Agents. Each Agent has endogenous and exogenous factors that impact on Trust decision making and this in turn affects the Trust relationship. In view of the Trust Model, we see that both endogenous factors and exogenous factors are related to Agents in the Trust Model.

Endogenous factors of an Agent refer to internal factors, psychological factors and personal characteristics, knowledge or skills etc of the Agent. The endogenous factors, including psychological factors, are factors internal to the Trusting parties. These internal factors can never be captured explicitly and they change as time passes. An Agent's "willingness" and "capability" are part of endogenous factors. When predicting Trust in a relationship, the factors that influence the Trust decision and that cannot be explicitly managed so far, are the endogenous factors. For example, if a person's thinking is changed; no one could know or capture this immediately. Endogenous factors cause the changes in the Trust relationship. However, the endogenous factors cannot be captured directly on hand, thus the 
measure and prediction of Trustworthiness of an Agent is only at an estimate or approximate level. The challenge of the Trustworthiness measure and prediction is that we are unable to explicitly capture the endogenous characters of Agents. Therefore, we have to develop some methods for the Trustworthiness measure and prediction that can through some external factors for which we can observe, capture and use it to give an estimate of the Trustworthiness of the Agent.

Exogenous factors are known as external factors of Trust, such as external activities, i.e. behavioural changes such as making a commitment to deliver a service or valuating an actual service delivery. These external activities can be identified and predicted. Exogenous activity influences the Trust Value and prediction; it may be caused by the environment where a business interaction is carried out or a service provider is unable to fulfil their commitments. The service oriented environment is a heterogeneous environment and consists of anonymous, pseudo-anonymous, and non-anonymous users or machines communicating with each other for services. In P2P service oriented networks, file sharing applications such as Gnutella and Napster enable the users to share files amongst each other. Free Net is a P2P oriented service network for anonymous storage. SETI@HOME is an example of a pseudo anonymous P2P application for distributed computing. In non-anonymous service oriented environments, such as Logistic networks, Agents make use of each other's resources. These resources can be either physical resources (like warehouse space or the transport capabilities) or digital resources (like each other's trackand-trace applications). However, the exogenous factors or external activity can be captured, analysed, measured and be calculated to determine a level of Trustworthiness. The challenge of the Trust measure and Trustworthiness prediction is to develop an estimation method that can handle heterogeneous environments and anonymous, pseudo-anonymous, and non-anonymous users and service providers and service interactions for predicting the Trustworthiness value.

\subsection{Reason for Fuzziness and Dynamism}

The six fuzzy and dynamic characteristics of Trust are triggered by Agents (Figure 1). As an Agent's endogenous factors are hard to capture and predict, and exogenous factors have a strong impact on the Agent's self development and Agent decision making. However, external factors can be captured and therefore can be used to help estimate an Agent's Trustworthiness. In view of the Trust Model (Figure 2), we see that both endogenous factors and exogenous factors are related to Peers in Trust relationships.

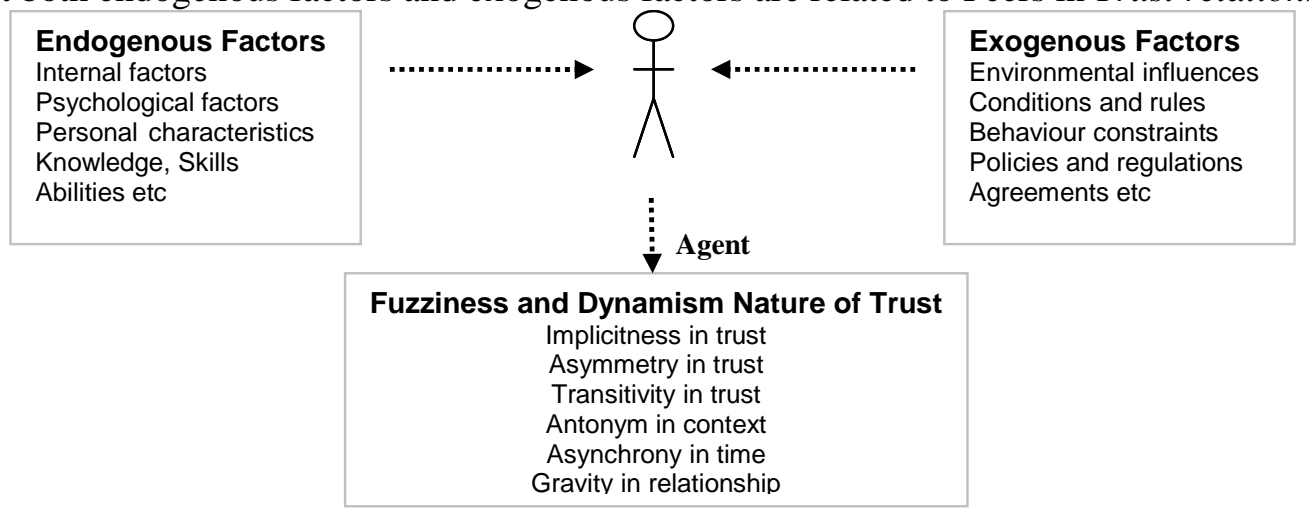

Figure 1 Exogenous and Endogenous factors of Agents [2]

\subsection{Fuzzy and Dynamic Characteristics and Trustworthiness Measure}

The dynamism of Trust is influenced by the factors associated with Trust and Trust Relationships. We now analyse eight characteristics of Trust aligned with the Trust Definition and Trust Model, in order to reason factors that determine the Trust dynamics.

While some changes (such as external behaviour) can be predicted, because they can be explicitly defined, others cannot be predicted (such as internal factors of Agents), because they cannot be explicitly defined. We can only give a measure or an estimate of the dynamism of Trust in the Trust relationship. We note that change can be caused by external factors as well as internal factors. In real life, we note that both factors could cause the change. However, the internal factors are hard to capture and predict, even 
with great scientific studies. This is unlike external factors, where one can feel them, predict them, and try to manage them. Therefore, the internal factors cause the dynamism or changes. These are the factors that humans or machines cannot manage. Humans or machines can manage the external factors that cause the dynamism or changes. They can be captured so they are considered to be static.

\section{$5 \quad$ Managing Trust}

There are several issues to be considered in the context of Managing Trust because Trust is dynamic and not always well-defined.

\subsection{Measuring the Service}

In most internet Trustworthiness systems, they have features on measuring service providers, merchants, or on-line shops. However, if a provider offers a very good service on books but very bad service on delivery, then the Trustworthiness value for the merchant should be distinguished. Measuring the service is most difficult task in the internet, or in the service oriented environment. It is important, that in the service oriented network environment, we should provider service rating, or Trustworthiness of service. Each service provider may have a number of services; however, the objective here is to measure the service of the provider, rather than that of the provider.

\subsection{Measuring the Product or Website}

This measuring has been used in most internet Trustworthiness systems. The features include measuring service providers, merchants, or on-line shops. The measure is much simpler than measuring the service, where human intelligence behind the service and have right to give input on the quality of service. Where in here, measuring the product, for example, if we say a camera is good or bad, we do not have to get 'camera' opinion about the comments that customer made.

\subsection{Managing the Dynamism of Trust}

In the existing literature the methods of managing Trust focus on assigning the Trust Value with the assumption that there is only one context and the Trust Value is assigned only for that context. This is due to the fact that many e-service providers only provide a single service (single context). However, this assumption becomes less relevant as the concept of e-services has expanded to multiple services over the last few years. Also in the literature, the methods of managing Trust only consider one Trust Value and the value does not change These methods do not consider the dynamic nature of Trust and the change of Trust Values with time.

\subsection{Time Spot, Time Slot and Time Space}

Trustworthiness prediction is defined as the process of determining the future Trust Value known as Trustworthiness value of the Trust ed entity or Agent, given it's past repute values or historical Trust Value or direct interaction from the given time spot, slot and space. A Time Spot is a particular time at which an entity interacted with another entity and subsequently assigned a Trustworthiness value to it. The Time Slot of a Trustworthiness prediction is the breadth or duration of time over which the Trust Value from the historical Trust Value or repute value is collected.

In order to analyse the dynamic behaviour of Trust Values, a time space consists of a number of nonoverlapping time slots. An entity will have a Trust Value or repute value for each time slot. These past Trust Values or repute values are aggregated and used for predicting future Trust Values known as a Trustworthiness value. The time space of a Trustworthiness prediction is the total duration of time over which the behaviour of the Trusted entity will be analysed and process of Trustworthiness prediction carried out. The repute value is a Trust Value for an entity, i.e., its reputation in a given context and in a given time slot as recommended by a witness entity or witness Agent.

\subsection{Managing the Trust Dynamism}


In order to manage Trust over the network as adequately as possible, one must consider the fuzziness and dynamism of the Trust. To give an estimate of a Trustworthiness value, we will carry out a correlation between an expected service and an actual delivered service to predict a Trustworthiness Value.

For the determination of the Trustworthiness value for a Trusted entity or Agent, we choose to apply a technique used in the human world, i.e. a correlation between an expected behaviour and an actual behaviour to determine the level of Trust. We adopt this approach for the Trustworthiness prediction in ebusiness or e-services to overcome the dynamism of Trust. The expected behaviour of the Trusted Agent is the mutually anticipated conduct of the Trusted Agent prior to its interaction with the Trusting Agent. The correlation is the degree of similarity between the expected delivery of the Trusted Agent and actual delivery of the Trusted Agent during interaction.

\subsection{Correlation of Behaviour}

Correlation refers to how similar the following two factors are: (1) The impression that the Trusting Agent has of the Trusted Agent in a given context, and (2) The outcome of the interaction between the Trusting Agent and the Trusted Agent in that particular context in a given timeslot. The greater the correlation between these two factors, the higher the Trustworthiness value assigned to the Trusted Agent by the Trusting Agent and vice versa. Strong correlations between the two factors indicate that the Trusted Agent met the impression held by the Trusting Agent, in that context. Conversely, a weak correlation indicates that the Trusted Agent did not meet the impression held by a Trusting Agent.

\subsection{Challenges in Trust Measure and Prediction}

In the existing literature the methods of managing Trust focus on assigning the Trust Value with the assumption that there is only one context and the Trust Value is assigned only for that context. This is due to the fact that many e-service providers only provide a single service (single context). However, this assumption becomes less relevant as the concept of e-services has expanded to multiple services over the last few years. Also in the literature, the methods of managing Trust only consider one Trust Value and that the value does not change. These methods do not consider the dynamic nature of Trust and the change of Trust Values with time.

Trustworthiness is a prediction of future Trust Values that depicts the level of the Trust Relationship that the Trusting Agent has with the Trust ed Agent in a given context, in a given time slot and with a given type of initial relationship association In other words, Trustworthiness is a prediction of the Trust level against context and time with the type of initiation of the Trust relationship. The prediction can only be done by correlating the actual behaviour with the expected behaviour, in a given context and in a particular time slot with respect to a given method of initiation. The constraint with the measurement of Trust and the prediction of Trustworthiness lies in the inability to handle the "internal factors" of Agents, namely their "willingness" and "capability".

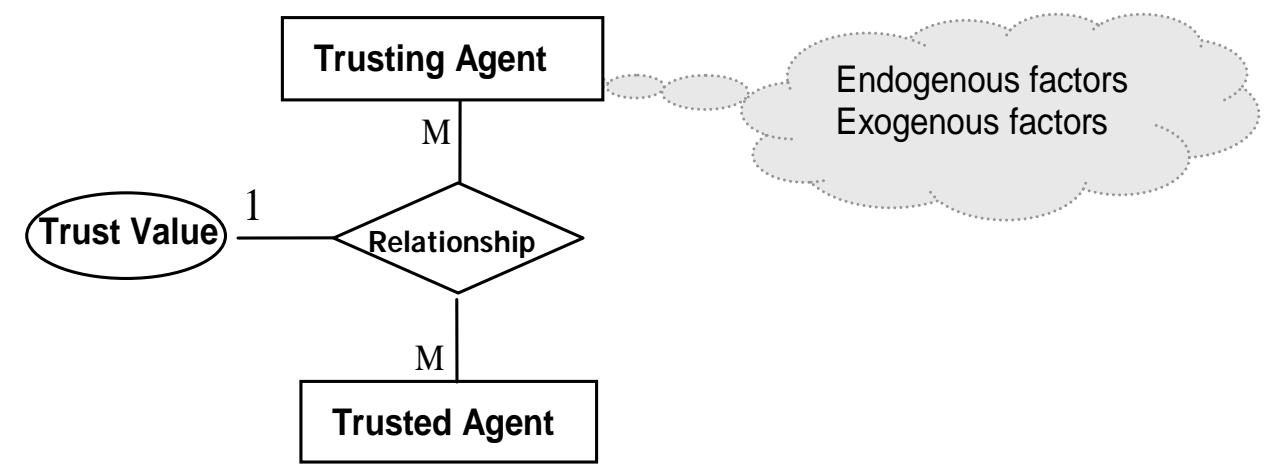

Figure 2 Alignment of the Agent's Endogenous and Exogenous Factors in the Trust Model [2].

In the Trust Model (Figure 2), we see that both endogenous factors and exogenous factors are related to Agents in the Trust Model. 


\begin{tabular}{|l|l|l|l|l|}
\hline $\begin{array}{l}\text { Trust Definition } \\
\text { Contains concepts }\end{array}$ & $\begin{array}{l}\text { Trust Model } \\
\text { Contains concepts }\end{array}$ & Trust Characteristics & $\begin{array}{l}\text { Agent } \\
\text { Characteristics }\end{array}$ & Trustworthiness \\
\hline Belief & $\begin{array}{l}\text { Implicitness of Trust } \\
\text { Asymmetry in Trust } \\
\text { Transitivity of Trust }\end{array}$ & Fuzzy result \\
\hline $\begin{array}{l}\text { Trusting Agent and } \\
\text { Trusted Agent }\end{array}$ & Relationship & $\begin{array}{l}\text { Gravity of } \\
\text { Relationship }\end{array}$ & Endogenous & Fuzzy result \\
\hline $\begin{array}{l}\text { Willingness } \\
\text { Capability } \\
\text { Delivery of the } \\
\text { mutually agreed } \\
\text { services }\end{array}$ & $\begin{array}{l}\text { Agents } \\
\text { (Conceptual behaviour) }\end{array}$ & $\begin{array}{l}\text { Agents } \\
\text { (External behaviour) }\end{array}$ & Exogenous & Explicit result \\
\hline A given Context & Context & Antonym in Context & & Explicit result \\
\hline A given Timeslot & Timeslot & $\begin{array}{l}\text { Asynchrony in Time } \\
\text { Space }\end{array}$ & Explicit result \\
\hline
\end{tabular}

Table 1 The Factors determining the Nature of Trust.

In the above Table 1, we note that: The 1 st column describes the concepts of Trust. The $2^{\text {nd }}$ column describes the Trust Model and all related concepts in the Trust Model (Figure 2). Column 2 is a pictorial representation of Column 1 (see Figure 2) [2]. The 3rd column aligns the fuzzy and dynamic characteristics to the trust definition and Trust Model. The $4^{\text {th }}$ column aligns the endogenous and exogenous (Internal and xternal) characteristics of an Agent to the trust definition and the Trust Model. The $5^{\text {th }}$ column shows that trustworthiness measure or prediction and what concepts can be explicitly defined and which concepts cannot.

In Table 2 below, we further explain what can be measured and what cannot. We note that change can be caused by external factors as well as internal factors. In real life, we note that both factors could cause the change. However, the internal factors are hard to capture and predict, even after intense empirical studies of a person's psychology. This is unlike external factors, where one can directly observe them, predict them, and try to manage them. Therefore, the internal factors cause the dynamism or changes and they are the factors that humans or machines cannot manage. Humans or machines can manage the external factors that cause the dynamism or changes, but they can be captured so they are considered to be stable in the Trust Model.

\begin{tabular}{|c|c|c|}
\hline Aspects of the Trust & Relation to Agents & Dynamism Analysis \\
\hline $\begin{array}{l}\text { Belief } \\
\text { Willingness } \\
\text { Capability }\end{array}$ & $\begin{array}{l}\text { Agent's } \\
\text { internal activity }\end{array}$ & $\begin{array}{l}\text { Internal factors are very hard to capture. They could be changed } \\
\text { by both internal influence and external influence. For example, } \\
\text { more education or an accident could change the capability of a } \\
\text { person, or psychological advice could affect the level of } \\
\text { willingness. However, no one can predict how much they will } \\
\text { change with time. Therefore, internal factors cause the dynamic } \\
\text { nature in the trust model. }\end{array}$ \\
\hline $\begin{array}{l}\text { Trusting Agent and } \\
\text { Trusted Agent }\end{array}$ & $\begin{array}{l}\text { Agent's } \\
\text { external activity }\end{array}$ & $\begin{array}{l}\text { Identifying a Trusting Agent or a Trusted Agent is an external } \\
\text { activity. They can be explicitly defined. }\end{array}$ \\
\hline $\begin{array}{l}\text { Deliver the mutually } \\
\text { agreed services }\end{array}$ & $\begin{array}{l}\text { Agent’s } \\
\text { external activity }\end{array}$ & $\begin{array}{l}\text { External factors can be captured though correlation of expected } \\
\text { delivery of the service compared to the actual service that is } \\
\text { provided. }\end{array}$ \\
\hline $\begin{array}{l}\text { A given Context, } \\
\text { A given Timeslot }\end{array}$ & $\begin{array}{l}\text { Agent's } \\
\text { external activity }\end{array}$ & $\begin{array}{l}\text { These are external factors and can be captured; therefore they } \\
\text { are not the cause of the dynamism in the Trust Model. }\end{array}$ \\
\hline
\end{tabular}

Table 2 Agent's internal and external factors, and the impact on the Dynamism of Trust

\section{Summary}

The limitation with the measurement of Trust and the prediction of Trustworthiness lies in the inability to consider accurately the internal factors of Agents, namely their willingness and capability. Since 
capability and willingness are by and large not directly observable, we arrive at an estimation of these by utilising the external factors (expected and actual behaviours) of Agents within the context of the relationship. In addition to the endogenic factors of willingness and capability, psychological factors of Trusting Agents contribute to Trust dynamism. The preference of the Trusting Agent for 'sensing' or 'intuition' will influence their decision to Trust a given Trusted Agent, with or without detailed information on the Trustworthiness of the Trust ed Agent. Whether a Trusting Agent gives preference to a 'thinking' or 'feeling' psychological disposition, will determine whether they make a decision based on facts or the personal values of the Trusted Agent.

We can conclude the following from our analysis of the dynamic nature of Trust:

- The internal factors of Agents determine the dynamic nature of the Agents

- The dynamic nature of the Agents leads to the dynamic nature of Trust, Trust relationships and Trust Values.

- "Context", "Time" and "Initiation of Relationship" are not dynamic as these factors are defined by the Agents, and once defined, they do not change.

\section{Acknowledgement}

The authors would like to thank you Miss Joy Bhattacharjya for help edit, compile and submit to TrustBus of the first draft paper.

\section{REFERENCES}

1. Burton, K.A., (2002), 'Design of the open privacy distributed reputation system', Available: [http://www.peerfear.org/papers/openprivacy-reputation.pdf] (10/11/2003).

2. Chang, E., Dillon T.S. Hussain F., 2005, Trust and reputation for Service Oriented Environments-Technologies for Building Business Intelligence and Consumer Confidence, John Wiley \& Sons, ISBN: 0-470- 01547-0

3. Chang, E., Talevski, A. \& Dillon, T., (2003), 'Web service integration in the extended logistics enterprise', Proceedings of the IEEE Conference on Industrial Informatics, INDIN, Banff, Canada.

4. Egger, N.F., (2000a), 'Trust me, I'm an online vendor', Towards a Model of Trust for E-Commerce System Design, Available: [http://www.zurich.ibm.com/ mrs/chi2000/contributions/egger.html] (10/09/2003).

5. Egger, N.F., (2000b), 'Towards a model of trust for e-commerce system design', Available: [http://www.zurich.ibm.com/ mrs/chi2000/contributions/egger.html] (29/05/2003).

6. Egger, N.F., (2003), 'Deceptive technologies: Cash, ethics and HCI', Available: [http://www.ecommuse.com/research/publications/sigchi_bulletin.htm] (23/05/2003).

7. Hussain, F., Chang, E. \& Dillon, T. S., (2004), 'Classification of trust relationships in peer-to-peer (P2P) communications', Proceedings of the Second International Workshop on Security in Information Systems WOSIS,

8. Kim, J. \& Moon, J.Y., (1997), 'Emotional usability of customer interfaces', Available: [http://hci.yonsei.ac.kr/non/e02/97-CHI Emotional_Usability_of_Customer_Interface.pdf] (23/08/2003).

9. http://www.gnutella.com/

10. http://www.napster.com/

11. Myers, S., (2003), 'Working out your Myers Briggs Type', Available: [http://www.teamtechnology.co.uk/tt/tarticl/mb-simpl.htm] (27/12/2003).

12. Rahman, A.A. \& Hailes, S., (2003a), 'Relying on trust to find reliable information', Available: http://www.cs.ucl.ac.uk/staff/F.AbdulRahman/docs/dwacos99.pdf] (7/08/2003).

13. Rahman, A.A. \& Hailes, S., (2003b), 'A distributed trust model', Available: http://citeseer.nj.nec.com/cache/papers/cs/ .../abdul-rahman97distributed.pdf] (5/09/2003).

14. Rahman, A.A. \& Hailes, S, (2003), 'Supporting trust in virtual communities', Available: [http://citeseer.nj.nec.com/cache/papers/cs...../abdul-rahman00supporting.pdf]

15. SETI@HOME, Available: [http://pwp.netcabo.pt/knology/SETI_ENG.htm] (28/09/2003)

16. Shelat, B. \& Egger, F,N., (2002), 'What makes people trust online gambling sites?' Available: [http://www.ecommuse.com/research/publications/chi2002.pdf] (10/08/2003).

17. Smith, J. H., (2002), 'The architectures of trust', University of Copenhagen.

18. Wang, Y. \& Vassileva, J., (2003), 'Trust and reputation model in peer-to-peer', Available: [www.cs.usask ca/grads/yaw181/ publications/120_wang_y.pdf] (15/10/2003). 